\title{
Relação entre macrofauna edáfica e atributos químicos do solo em diferentes agroecossistemas
}

\author{
Sandra Santana de Lima(1), Adriana Maria de Aquino(2), Luiz Fernando Carvalho Leite ${ }^{(3)}$, \\ Elena Velásquez ${ }^{(4)}$ e Patrick Lavelle ${ }^{(5)}$
}

${ }^{(1)}$ Universidade Federal Rural do Rio de Janeiro, Departamento de Agronomia, BR 465, Km 7, CEP $23890-000$ Seropédica, RJ.
E-mail: sandra.biologa@hotmail.com (2)Embrapa Agrobiologia, Avenida Alberto Braune, no 223, CEP 28613-001 Nova Friburgo,
RJ. E-mail: adriana@cnpab.embrapa.br ${ }^{(3)}$ Embrapa Meio Norte, Avenida Duque de Caxias, no 5.650, CEP 64006-220 Teresina, PI.
E-mail: luizf@cpamn.embrapa.br (4)Universidad Nacional de Colômbia, Carrera 30, no 45-03, Bogotá, Colômbia. E-mail: evelasquezi@unal.edu.co
${ }^{(5}$ Institut de Recherche pour le Développement, 32, Avenue Henri Varagnat, 93143 Bondy, France. E-mail: patrick.lavelle@bondy.ird.fr

Resumo - O objetivo deste trabalho foi avaliar o efeito do uso do solo sobre a densidade e a diversidade da macrofauna invertebrada, bem como a relação dessa com atributos químicos do solo em diferentes agroecossistemas. Foram estudados cinco agroecossistemas: sistema ecológico com três anos de adoção (SE3), sistemas agroflorestais com seis (SAF6) e dez (SAF10) anos de adoção; agricultura de corte e queima (ACQ), e floresta nativa (FN). Em cada sistema, foram coletadas aleatoriamente cinco amostras solo, em forma de blocos $(25 \times 25 \mathrm{~cm})$, na profundidade de $10 \mathrm{~cm}$, nas épocas seca (outubro, 2006) e chuvosa (maio, 2007). A relação entre os atributos químicos e a macrofauna edáfica, nos diferentes sistemas de uso do solo, foi determinada por meio da análise da coinércia. Maior abundância da macrofauna foi observada na época chuvosa. Os sistemas SE3, SAF6 e SAF10 apresentaram maior riqueza de espécies e índices de Shannon e Pielou, independentemente da época de coleta. O manejo agroflorestal favoreceu a ocorrência de "engenheiros do ecossistema". Os sistemas agroflorestais propiciam melhores características químicas do solo e aumentos na abundância e riqueza da macrofauna invertebrada do solo.

Termos para indexação: agricultura de queima e corte, invertebrados do solo, sistemas agroflorestais, sistemas de uso do solo, transição cerrado-floresta.

\section{Relationship between edaphic macrofauna and soil chemical attributes in different agroecosystems}

\begin{abstract}
The objective of this study was to evaluate land use effects on the abundance and diversity of invertebrate macrofauna and its relationship with the soil chemical characteristics in different agroecosystems. Five systems were studied: ecological based system at three years of adoption (ES3), agroforestry systems at six (AFS6) and ten years of adoption (AFS10), slash and burn agriculture (SBA), and native forest (NF). In each system, five samples were collected in the form of soil monoliths $(25 \times 25 \mathrm{~cm})$ at $10 \mathrm{~cm}$ depth, during the dry (October 2006) and rainy seasons (May 2007). The relationship between edaphic macrofauna and chemical attributes in the different land use systems was determined by coinertia analysis. The highest abundance of macrofauna was observed in the rainy season. Systems SE3, SAF6 and SAF10 showed higher species richness and indices of Shannon and Pielou, regardless of the sampling season. The agroforestry management favored the occurrence of "ecosystem engineers". Agroforestry systems provide better soil chemical characteristics and increase soil invertebrate macrofauna abundance and richness.
\end{abstract}

Index terms: slash and burn agriculture, soil invertebrates, agroforestry systems, land use systems, savannaforest transition.

\section{Introdução}

A fauna edáfica representa uma força motriz na decomposição e ciclagem dos nutrientes (Höfer et al., 2001). Ela ocupa diversos níveis tróficos dentro da cadeia alimentar no solo e afeta a produção primária de maneira direta e indireta (Aquino et al., 2008a). A macrofauna edáfica é representada por animais com diâmetro do corpo maior do que $2 \mathrm{~mm}$, como formigas, coleópteros, aranhas, minhocas, centopéias, térmitas, diplópodes, etc (Aquino et al., 2008b). Alguns grupos

Pesq. agropec. bras., Brasília, v.45, n.3, p.322-331, mar. 2010 
de animais são responsáveis pela predação de outros invertebrados e outros contribuem diretamente na modificação da estrutura do solo, por meio de sua movimentação pelo perfil (Correia \& Andrade, 2008), sendo por isso comumente denominados de engenheiros do solo (Lavelle et al., 1997).

Os invertebrados do solo alteram as populações e atividade de microrganismos responsáveis pelos processos de mineralização e humificação da matéria orgânica do solo e, portanto, exercem influência sobre a disponibilidade de nutrientes assimiláveis pelas plantas (Decaëns et al., 2003). A composição da comunidade da macrofauna edáfica do solo e sua abundância são indicadores da biodiversidade do solo e da intensidade das atividades biológicas (Velásquez et al., 2007). No entanto, os efeitos dos organismos do solo sobre os processos dos ecossistemas raramente são aparentes em virtude da escala em que as medições são feitas, comumente incapazes de representar grandes áreas, e dos curtos intervalos de tempo normalmente pesquisados. Dessa forma, esses processos são mais frequentemente relacionados a outras variáveis, como vegetação, propriedades do solo ( $\mathrm{pH}$, mineralogia etc.) e, principalmente, clima (Anderson, 2009).

A agricultura intensiva envolve elevado uso de insumos externos, que promovem alterações importantesna estrutura das comunidades, abundâncias e biomassas da fauna e microbiota do solo (Marchão et al., 2009) e sobrepõem-se a processos biológicos do solo na definição da produtividade agrícola (Anderson, 2009). Contudo, para manutenção da qualidade do solo e da sustentabilidade de seu uso é fundamental que a abundância e diversidade de espécies da macrofauna edáfica seja promovida (Vohland \& Schroth, 1999; Merlim et al., 2005; Silva et al., 2006; 2008).

As alterações causadas pela atividade agrícola podem ocorrer indiretamente, pela melhoria na fertilidade do solo e aumento da qualidade nutricional dos resíduos, que possibilitariam colonização de habitats antes desfavoráveis. Também podem ocorrer diretamente pelo efeito negativo do revolvimento do solo e da aplicação de defensivos agrícolas (Marchão, 2007). Nesse sentido, sistemas conservacionistas, como os sistemas agroflorestais (SAFs), em virtude da semelhança com sistemas naturais (Barros et al., 2008), podem representar a combinação ideal para a biologia do solo, pela oferta de refúgio e a alta disponibilidade de matéria orgânica, para macro e microrganismos, sem que haja grandes perturbações advindas de manejo intensivo (Luizão \& Schubart, 1987), e podem até mesmo favorecer o restabelecimento da fauna do solo e dos diversos benefícios decorrentes da atividade desses organismos ao sistema.

Para ampliar o entendimento da abundância e da diversidade dos grupos da fauna e sua relação com as características químicas, físicas e de manejo do solo, tem sido comum o emprego de técnicas de análise multivariada (Barros et al., 2002; Alves et al., 2006; Aquino et al., 2008; Santos et al., 2008; Marchão et al., 2009; Nunes et al., 2009). A utilização dessas técnicas pode adicionar informações relevantes ao entendimento da variação conjunta de variáveisresposta, e facilitar a interpretação de dados biológicos (Coimbra et al., 2007).

O objetivo deste trabalho foi avaliar o efeito do uso do solo sobre a densidade e a diversidade da macrofauna invertebrada, e a relação desta com atributos químicos do solo em diferentes agroecossistemas.

\section{Material e Métodos}

O estudo foi realizado nos anos de 2006 e 2007, na comunidade Vereda dos Anacletos, localizada no município de Esperantina (03⒌ $54^{\prime} 07^{\prime \prime} \mathrm{S}, 42^{\circ} 14^{\prime} 02^{\prime \prime} \mathrm{W}$, altitude de $59 \mathrm{~m}$ ), região norte do Estado do Piauí. O clima da região é tropical subúmido, segundo a classificação de Köppen, com duas estações bem definidas, uma chuvosa e outra seca, com temperaturas anuais médias mínimas de $26^{\circ} \mathrm{C}$ e máximas de $34^{\circ} \mathrm{C}$. A precipitação média anual é de $1.400 \mathrm{~mm}$ e os maiores índices pluviométricos são concentrados de janeiro a maio (Figura 1). O solo é classificado como Argissolo Vermelho-Amarelo distrófico, textura franco-arenosa (Santos et al., 2006).

Para a realização deste trabalho, foram utilizadas áreas de um hectare de cinco sistemas de manejo: sistema de manejo ecológico, denominação atribuída em virtude da ausência do componente arbóreo nesse sistema e pela opção ao manejo com base ecológica, sem queima, revolvimento do solo e adição de insumos químicos com três anos de adoção (SE3); sistemas agroflorestais com seis (SAF6) e dez (SAF10) anos de adoção; área manejada com agricultura de corte e queima ( $\mathrm{ACQ}$ ); floresta nativa (FN), como referência da vegetação natural local. A região é caracterizada 
por apresentar uma formação vegetal predominante de transição entre cerrado e floresta secundária mista, possuindo extensas áreas com palmeira-babaçu [Orrbignya speciosa (Mart.) Barb. Rodr].

No SE3, a prática de corte e queima da vegetação foi utilizada até o ano de 2003. A partir de 2004, após a regeneração natural da vegetação, o manejo foi realizado apenas com duas roçadas: a primeira no final da época seca, tendo-se deixado o material vegetal sobre o solo; e a segunda, na época chuvosa, para que se garantisse o crescimento e desenvolvimento das espécies plantadas. Nesse sistema, eram cultivados milho (Zea mays L.) e mandioca (Manihot esculenta Crantz.), associados a espécies como mamão (Carica papaya L.), caju (Anacardium occidentale L.), melancia (Citrullus vulgaris Schrad.), maxixe (Cucumis anguria L.) e banana (Musa paradisiaca L.). As espécies frutíferas estavam distribuídas de forma aleatória na área, sendo que a maior proporção era representada pelo caju.

No SAF6, a área foi mantida com agricultura de corte e queima até 2001. Em 2002, essa prática foi substituída pela roçada da vegetação secundária, excluindo-se algumas espécies arbóreas. Foram realizadas duas roçadas: a primeira no final da época seca, em novembro, com o material proveniente do manejo tendo sido utilizado como cobertura do solo; e a segunda na época chuvosa, em fevereiro, para permitir o crescimento e desenvolvimento das espécies plantadas. Além disso, foi adicionada ao solo palha de carnaúba como cobertura. Foram cultivados nesse

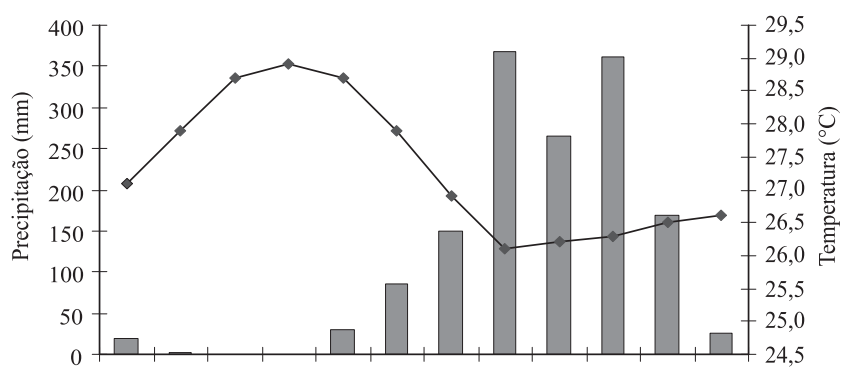

Jul. Ago. Set. Out. Nov. Dez. Jan. Fev. Mar. Abr. Maio Jun. Meses

$$
\begin{gathered}
\text { Meses } \\
\square \text { Precipitação } \longrightarrow \text { Temperatura }
\end{gathered}
$$

Figura 1. Precipitação pluvial e temperatura média mensal referente ao segundo semestre de 2006 e primeiro semestre de 2007, no município de Esperantina, PI. Fonte: Gerência de Hidrometeorologia da Secretaria do Meio Ambiente e Recursos Hídricos do Estado do Piauí. sistema: milho e algodão (Gossypium herbaceum L.) associados a caju, banana, amendoim [Arachis sylvestris (A.Chev.) A.Chev.], pinha (Annona squamosa L.), mamona (Ricinus communis L.), jatobá(Hymenaea sp.), jurubeba (Solanum sp.), pau-d'arco-amarelo (Tabebuia serratifolia) e carnaúba (Copernicia prunifera Mill.) e mandacaru (Cereus jamacaru DC.). O arranjo espacial das espécies arbóreas nativas e frutíferas foi aleatório. A espécie Anacardium occidentale predominava sobre as frutíferas.

$\mathrm{Na}$ área com SAF10, o manejo convencional com uso de corte e queima foi substituído pelo manejo ecológico em 1997. O material vegetal decorrente da roçada, que anteriormente era queimado, passou a servir como cobertura do solo. A partir de então, foram introduzidas espécies frutíferas e, paralelamente, permitiu-se a regeneração natural de plantas pioneiras, as quais foram podadas antes das roçadas da época chuvosa. Além disso, foi adicionado ao solo esterco de caprinos. Nesse sistema, cultivou-se milho, abóbora (Curcubita pepo L.), fava (Phaseolus lunatus L.), mandioca, batata-doce [Ipomoea batatas (L.) Lam.] e algodão associados à acerola (Malpighia glabra L.), mamão, goiaba (Psidium guajava L.), banana, caju, manga (Mangifera indica L.), pinha, pitomba (Talisia esculenta Raldlk.), mamona, urucum (Bixa orellana L.), gergelim (Sesamum indicum L.), paud'arco, babaçu (Attalea speciosa Mart. ex Spreng.), gonçalo-alves (Astromiun fraxinifolium Schott), tamboril (Enterolobium sp.), jatobá, aroeira (Myracrodruon urundeuva Allemão), mufumbo (Combretum sp.) e unha-de-gato (Mimosa sp.). As espécies arbóreas e frutíferas foram distribuídas de forma aleatória, com maior densidade das plantas de caju, com uma distribuição de cerca de quarenta plantas por hectare, além de mamona, banana e manga, em menor proporção.

$\mathrm{Na}$ área de ACQ, o manejo do corte e queima da vegetação da floresta nativa ocorreu em 2005. Em 2006, a área foi cultivada com milho. A floresta nativa (FN) foi caracterizada por apresentar uma vegetação de floresta semidecídua preservada, onde eram observadas espécies de Cerrado e Caatinga, o que caracteriza uma área transicional. Não houve aplicação de fertilizantes químicos ou calagem em nenhum desses dois sistemas.

Foram realizadas duas coletas da macrofauna invertebrada do solo, uma em outubro de 2006, 
na época seca, e outra em maio de 2007, na época chuvosa (Figura 1). As amostragens foram realizadas utilizando-se o método recomendado pelo Programa "Tropical Soil Biology and Fertility" (TSBF), descrito por Anderson \& Ingram (1993). Em cada área, foram coletados cinco monólitos de solo, com o auxílio de um gabarito com $25 \times 25 \mathrm{~cm}$, a $10 \mathrm{~cm}$ de profundidade, distanciados 10 metros entre si, ao longo de um transecto determinado ao acaso. As amostras de solo foram acondicionadas em sacos de plástico, para posterior coleta dos indivíduos da macrofauna. Nos pontos de amostragem da fauna, também foram coletadas amostras de solo para a caracterização química $(\mathrm{pH}, \mathrm{Al}, \mathrm{Ca}, \mathrm{Mg}, \mathrm{K}, \mathrm{P}, \mathrm{N}$, $\mathrm{C}_{\text {org }}$ ) conforme Claessen (1997). Os resultados das análises químicas do solo sob os sistemas estudados são apresentados na Tabela 1 .

A triagem das amostras foi realizada manualmente, com a coleta de todos os indivíduos com mais que $10 \mathrm{~mm}$ de comprimento ou com diâmetro corporal superior a $2 \mathrm{~mm}$, que foram armazenados em solução de álcool a $70 \%$. A identificação e a contagem foram efetuadas com auxílio de lupa binocular. Os indivíduos foram classificados, conforme a classe e ordem, e separados de acordo com o estádio de desenvolvimento em adultos ou imaturos (larvas).

A densidade da macrofauna edáfica foi obtida a partir da transformação do número de indivíduos, encontrados em cada amostra, em número de indivíduos por metro quadrado (indivíduos $\mathrm{m}^{-2}$ ). Para cada área, foram calculados: o índice de diversidade de Shannon $(\mathrm{H})$, que considera a riqueza de espécies (S) e sua abundância relativa $\left(p_{i}\right)$, definido por $H=-\sum p_{i} \ln p_{i}$; índice de equitabilidade de Pielou (e), que refere-se ao padrão de distribuição dos indivíduos entre as espécies, definido por e $=\mathrm{H} / \mathrm{ln} \mathrm{S}$. Além disso, a riqueza média $(\mathrm{RM})$ representa o número médio de espécies da fauna coletados por amostra, em cada tratamento (Dias et al., 2006). Os valores do total de indivíduos nas duas épocas de coletas foram comparados pelo teste de Tukey a $5 \%$ de probabilidade, por meio do programa Sisvar (Ferreira, 2008).

Com auxílio do programa ADE-4 (Thioulouse et al., 1997), foram realizadas análises multivariadas de componentes principais (ACP) relativas à macrofauna edáfica e às variáveis químicas do solo ( $\mathrm{pH}, \mathrm{Al}, \mathrm{Ca}, \mathrm{Mg}, \mathrm{K}, \mathrm{P}, \mathrm{N}, \mathrm{C}_{\text {org }}$ ). Utilizouse o método de coinércia (AC) para determinar correlações entre os autovetores e as variáveis avaliadas. A significância estatística das correlações entre as variáveis ambientais e as da macrofauna foi avaliada pelo teste de permutação de Monte Carlo (Ter Braak, 1995).

\section{Resultados e Discussão}

Em ordem decrescente de densidade de indivíduos, os maiores níveis taxonômicos observados foram: Isoptera, Coleoptera (larvas), Formicidae, Pseudoscorpiones, Chilopoda, Blattodea e Thysanura, na época seca; e Isoptera, Formicidae, Oligochaeta, Isopoda, Araneae, Coleoptera, Diplopoda, Chilopoda, Coleóptera (larvas), Diplura, Pseudoscorpiones e Hemíptera, na época chuvosa.

A maior densidade foi observada na época chuvosa (5.498 indivíduos $\left.\mathrm{m}^{-2}\right)$. $\mathrm{O}$ número de indivíduos da macrofauna nos sistemas SE3, SAF6 e SAF10 diferiu significativamente entre as épocas de coleta (Tabela 2). Esse resultado está relacionado à grande sensibilidade da maior parte das espécies da macrofauna observadas às condições climáticas (Nunes et al., 2008). As menores densidades foram observadas na época seca, com exceção de ACQ e FN, que apresentaram maior estabilidade entre épocas de

Tabela 1. Características químicas, na época chuvosa, de um Argissolo Vermelho-Amarelo distrófico, na camada de 0-10 cm, em sistemas de uso de solo com base ecológica com três anos de adoção (SE3), agroflorestais com seis (SAF6) e dez anos de adoção (SAF10), sistemas baseados em agricultura de corte e queima (ACQ), e em floresta nativa (FN).

\begin{tabular}{|c|c|c|c|c|c|c|c|c|}
\hline \multirow[t]{2}{*}{ Sistema } & \multirow{2}{*}{$\begin{array}{c}\mathrm{pH} \mathrm{em} \\
\mathrm{H}_{2} \mathrm{O}\end{array}$} & COT & NT & $\mathrm{Ca}$ & $\mathrm{Mg}$ & K & \multirow{2}{*}{$\begin{array}{c}\mathrm{P} \\
\left(\mathrm{mg} \mathrm{dm}^{-3}\right)\end{array}$} & \multirow{2}{*}{$\begin{array}{c}\mathrm{Al} \\
\left(\mathrm{cmol}_{\mathrm{c}} \mathrm{dm}^{-3}\right)\end{array}$} \\
\hline & & \multicolumn{2}{|c|}{ 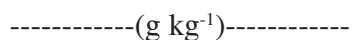 } & ----------- & $\mathrm{nol}_{\mathrm{c}} \mathrm{dn}$ & --------- & & \\
\hline SE3 & 5,8 & 16,0 & 1,2 & 3,43 & 0,59 & 0,13 & 5,10 & 0,16 \\
\hline SAF6 & 5,6 & 35,4 & 2,5 & 7,35 & 1,81 & 0,38 & 13,98 & 0,16 \\
\hline SAF10 & 6,0 & 32,7 & 2,2 & 15,47 & 1,68 & 0,29 & 16,85 & 0,16 \\
\hline ACQ & 5,7 & 12,2 & 1,3 & 3,15 & 0,36 & 0,13 & 8,79 & 0,16 \\
\hline $\mathrm{FN}$ & 4,6 & 31,5 & 1,9 & 1,20 & 0,95 & 0,21 & 2,46 & 1,64 \\
\hline
\end{tabular}




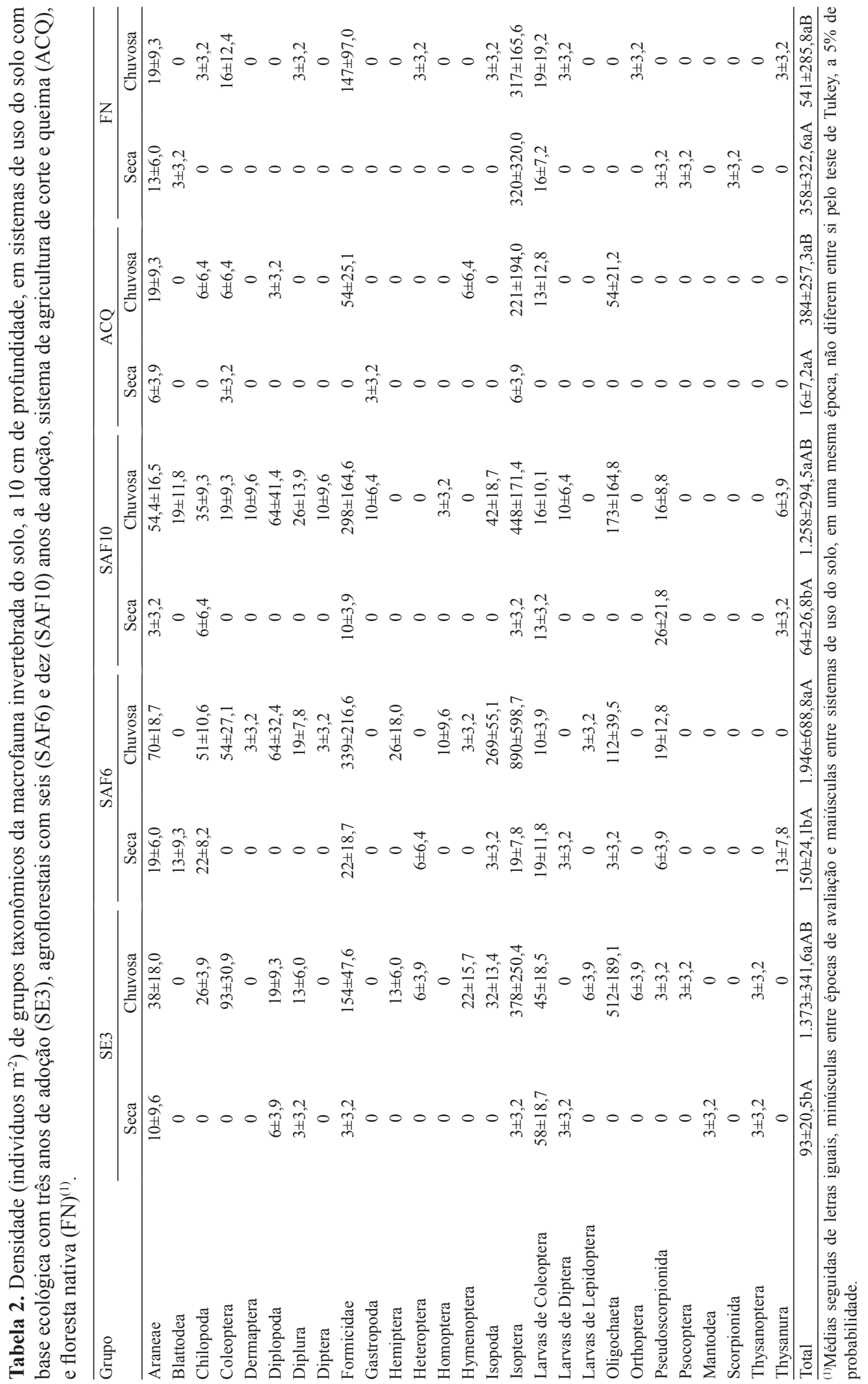


amostragem. Esses sistemas, no entanto, apresentaram densidades menores que as observadas nos outros sistemas na época

A ocorrência de formigas, térmitas e minhocas foi elevada em todos os sistemas, especialmente nos SAF6 e SAF10 (Tabela 2), provavelmente em virtude do ambiente coberto e do manejo com a poda e roçada que promovem melhores condições climáticas e de disponibilidade de alimentos. Esses grupos são denominados "engenheiros do ecossistema", em razão de seus efeitos sobre a estruturação do solo, com criação de estruturas biogênicas, que podem ser galerias, ninhos, câmaras e bolotas fecais, e que podem afetar significantemente propriedades físicas dos solos, bem como a disponibilidade de recursos para outros organismos (Wolters, 2000; Anderson, 2009).

Os sistemas SE3, SAF6 e SAF10 apresentaram maiores valores dos índices de Shannon e Pielou e maior riqueza de espécies, independentemente da época de coleta (Tabela 3 ). O manejo desses sistemas favorece a melhor cobertura do solo, com aporte de restos das culturas e do material das podas, no caso dos SAFs. De acordo com Silva et al. (2007), a presença de cobertura vegetal no solo contribui para o aumento da disponibilidade de energia e promove a criação de novos habitats favoráveis à colonização por organismos invertebrados, o que pode beneficiar a sustentabilidade ecológica dos sistemas de produção. Na época chuvosa, houve decréscimo no valor do índice de Shannon no SAF6, ocasionado pela maior concentração de indivíduos das famílias Formicidae e Isoptera, principalmente (Tabelas 2), mas sem dominância de nenhum deles. De acordo com Barros et al. (2008), a ausência de uma clara dominância entre essas espécies somente ocorre em razão dos aportes orgânicos serem diversificados, o que permite a colonização por diferentes organismos (Barros et al., 2008). Isso ocorreu principalmente nos sistemas agroflorestais.

As práticas agrícolas de manejo do solo provocam impacto e resultam em redução na densidade e riqueza dos organismos da macrofauna do solo (Silva et al., 2006). Nesse sentido, a implementação de sistemas agroflorestais favoreceu o aumento na riqueza e densidade de espécies da macrofauna edáfica (Tabela 3), já que os maiores valores dessas variáveis foram observados nesses sistemas. A riqueza de espécies variou de 3 a 18, entre os sistemas estudados. Amplitude similar foi observada em regiões de Cerrado por Aquino et al. (2008b).

Em contraste à abundância e riqueza de espécies observada nos SAFs, o sistema ACQ apresentou redução drástica nesses valores (Tabela 3). Este resultado está relacionado à redução do aporte de resíduos culturais pela queima, o que reduz a disponibilidade de alimentos e de nichos para abrigo de espécies. Resultado semelhante foi observado por Nunes et al. (2009), ao estudarem o impacto da queima tradicional para o preparo do solo em áreas de Caatinga. Esses autores concluíram que o manejo do solo por meio de queimadas contribui

Tabela 3. Índices ecológicos relativos à macrofauna do solo na profundidade de $10 \mathrm{~cm}$, em sistemas de uso de solo com base ecológica com três anos de adoção (SE3), agroflorestais com seis (SAF6) e dez anos de adoção (SAF10), sistemas baseados em agricultura de corte e queima (ACQ), e floresta nativa (FN).

\begin{tabular}{|c|c|c|c|c|c|}
\hline Sistemas & Indivíduos $\mathrm{m}^{-2}$ & Índice de Shannon & Índice de Pielou & Riqueza média & Riqueza total \\
\hline & \multicolumn{5}{|c|}{ Época seca } \\
\hline SE3 & $92,8 \pm 20,5$ & 2,04 & 0,64 & 2,60 & 9 \\
\hline SAF6 & $150,4 \pm 24$ & 3,30 & 0,92 & 5,40 & 12 \\
\hline SAF10 & $64,0 \pm 26,7$ & 2,38 & 0,85 & 2,60 & 7 \\
\hline ACQ & $16,0 \pm 7,1$ & 0,27 & 0,17 & 0,80 & 3 \\
\hline \multirow[t]{2}{*}{$\mathrm{FN}$} & $358,4 \pm 322,5$ & 0,70 & 0,27 & 2,00 & 6 \\
\hline & \multicolumn{5}{|c|}{ Época chuvosa } \\
\hline SE3 & $1.372,8 \pm 341,6$ & 2,68 & 0,64 & 10,20 & 18 \\
\hline SAF6 & $1.945,6 \pm 688,8$ & 2,56 & 0,63 & 9,80 & 17 \\
\hline SAF10 & $1.257,6 \pm 294,5$ & 2,87 & 0,69 & 10,00 & 18 \\
\hline ACQ & $384,0 \pm 257,3$ & 1,99 & 0,63 & 3,80 & 9 \\
\hline $\mathrm{FN}$ & $540,8 \pm 285,8$ & 1,76 & 0,49 & 4,20 & 12 \\
\hline
\end{tabular}


para a redução da diversidade da macrofauna edáfica e culmina com a eliminação de alguns grupos taxonômicos.

O solo sob floresta nativa, em ambas as épocas de coleta, apresentou os menores valores dos índices de Shannon e Pielou, assim como baixos valores de riqueza (Tabela 3). Os menores índices estiveram associados à maior densidade de indivíduos do grupo Isoptera (Tabela 2). Nesse sistema, os maiores valores desses índices foram observados na época chuvosa. Esses resultados diferem dos observados por Silva et al. (2006), em áreas de Cerrado, que observaram maior riqueza na vegetação nativa do que em sistemas cultivados.

A análise de componentes principais (ACP) revelou que os dois primeiros eixos explicaram $40 \%$ da variabilidade total dos dados, com observação de registros entre 22 e $18 \%$ para o primeiro e segundo eixos, respectivamente. (Figura 2). O eixo 1 foi influenciado pelos grupos Isoptera, Diplopoda, Scorpionida, que apresentaram autovetores positivos. O eixo 2, por sua vez, foi influenciado pelos grupos Oligochaeta, Thysanura, com autovetores positivos, e pelos grupos Pseudoescorpionada, larva de Díptera, Formicidae e Coleóptera, que apresentaram autovetores negativos. $\mathrm{O}$ diagrama de ordenação mostra o agrupamento dos pontos em função dos sistemas de manejo do solo, em que os dois primeiros eixos explicaram 39,6\% da variação: $22 \%$ explicada pelo primeiro eixo, que sofreu maior influência dos sistemas SAF10 e SAF6; e 17,6\% pelo segundo, com influência dos demais sistemas de uso. Esse resultado evidencia clara separação dos sistemas (Figura 2).

Ao analisar as variáveis químicas do solo por meio da análise de componentes principais, observou-se que os dois primeiros eixos explicaram $78,6 \%$ da variância (Figura 3). O segundo eixo separou solos com maior concentração de bases e matéria orgânica dos solos com alta saturação de alumínio e baixa saturação de bases. O diagrama de ordenação em função do manejo dos sistemas de

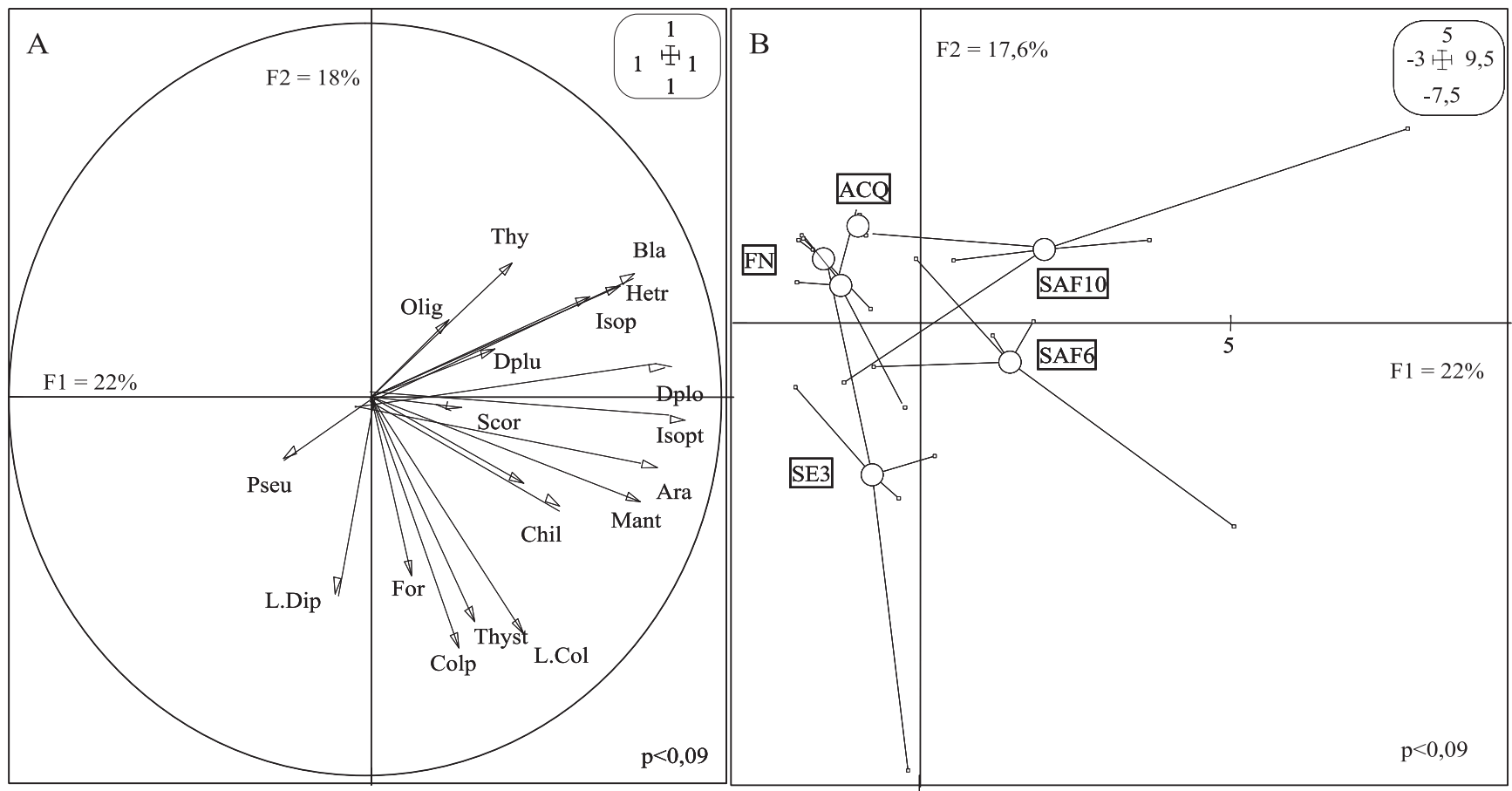

Figura 2. Análise dos componentes principais para a abundância e riqueza de espécies da macrofauna edáfica. A, círculo de correlações entre grupos taxonômicos da macrofauna: Olig, Oligochaeta; Thy, Thysanura; Bla, Blattodea; Hetr, Heteroptera; Isop, Isopoda; Dplo, Diplopoda; Scor, Scorpionida; Isopt, Isoptera; Chil, Chilopoda; Mant, Mantodea; Ara, Araneae; L.Col, larva de Coleoptera; L.Dip, larva de Diptera; Thyst, Thysanoptera; Colp, Coleoptera; For, Formicidae; Pseu, Pseudoescorpionada. B, diagrama de ordenação das amostras, em função do sistema de uso do solo: SE3, sistema de uso do solo com base ecológica com três anos de adoção; SAF6 e SAF10, sistemas agroflorestais com seis e dez anos de adoção, respectivamente; ACQ, agricultura de corte e queima; e FN, floresta nativa. p, significância estatística dos agrupamentos. 


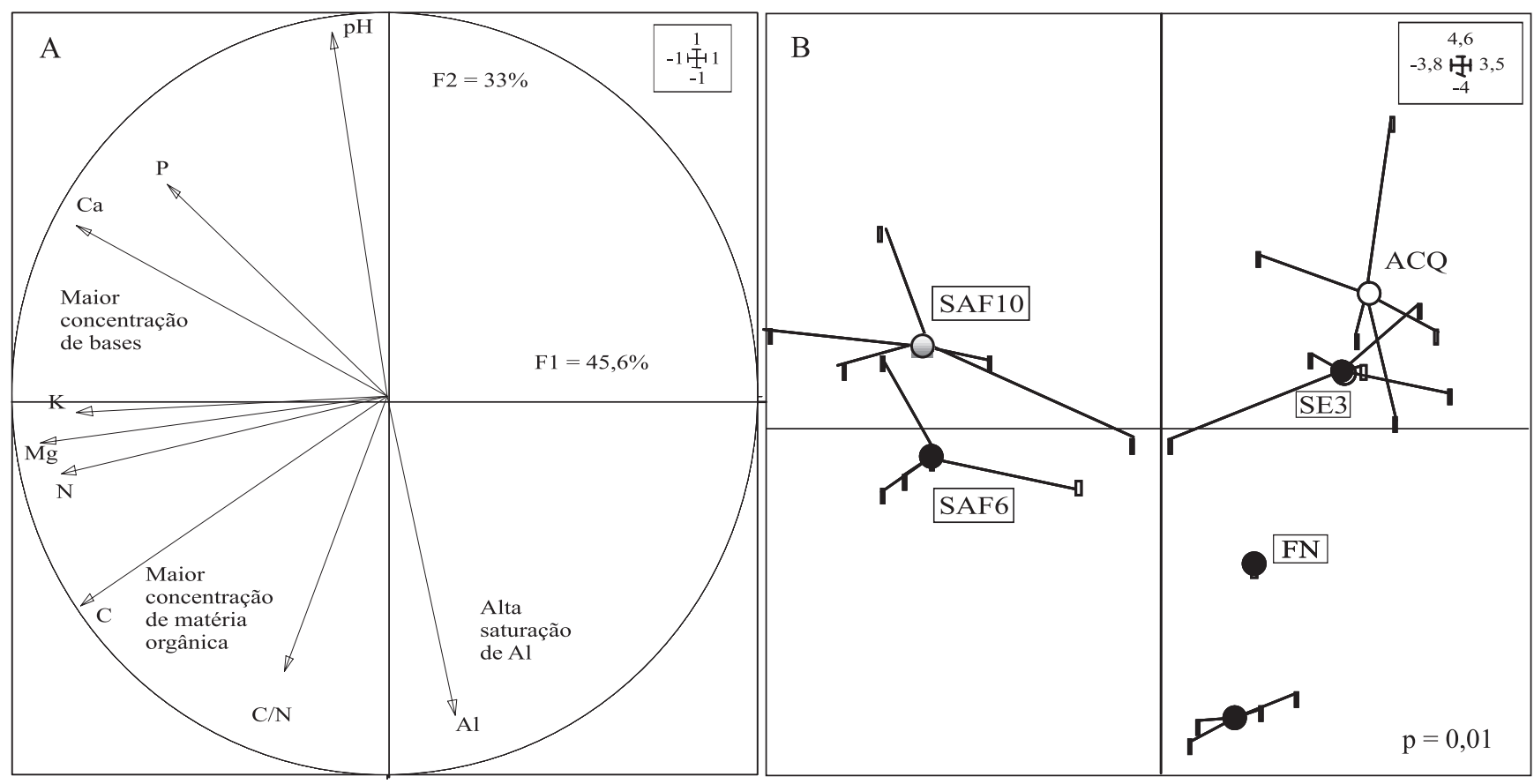

Figura 3. Análise dos componentes principais para as variáveis químicas do solo. A, círculo de correlações entre as variáveis químicas do solo. B, diagrama de ordenação das amostras, em função do sistema de uso do solo: SE3, sistema de uso do solo com base ecológica com três anos de adoção; SAF6 e SAF10, sistemas agroflorestais com seis e dez anos de adoção, respectivamente; ACQ agricultura de corte e queima; e FN, floresta nativa. p, significância estatística dos agrupamentos.

uso do solo mostra que o agrupamento dos sistemas foi influenciado pelas características químicas do solo $(\mathrm{p}<0,01)$. As melhores condições químicas do solo sob os SAF6 e SAF10 resultou no agrupamento desses sistemas, enquanto a diferença de manejo e menores teores de nutrientes os separou dos demais sistemas.

A análise da coinércia detectou correlação significativa entre os atributos químicos do solo e a macrofauna invertebrada do solo, sob os diferentes sistemas de manejo, e evidenciou similaridades entre os sistemas agroflorestais, com seu agrupamento resultante da melhoria nas características químicas e da abundância e riqueza da macrofauna invertebrada (Figura 4). Pelo teste de permutação de Monte Carlo, observou-se que os parâmetros utilizados para explicar os agrupamentos foram significativos $(p<0,01)$. Quando o nível de macronutrientes do solo aumentou, a abundância da macrofauna foi favorecida e a influência dos fatores químicos do solo para explicar os agrupamentos aumentou. Resultados semelhantes foram reportados por Barros et al. (2002) e Merlim et al. (2005).

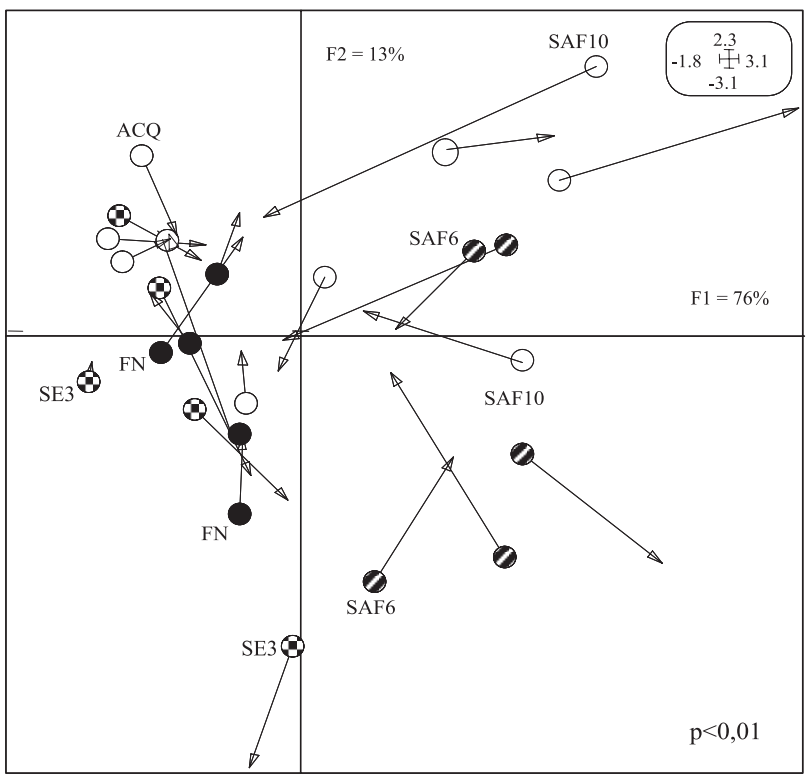

Figura 4. Análise de coinérica com distribuição dos sistemas de uso do solo (SE3 sistema com base ecológica de três anos, SAF6 e SAF10 sistemas agroflorestais com seis e dez anos de adoção, respectivamente, ACQ agricultura de corte e queima e FN e floresta nativa) em relação às variáveis de macrofauna e química do solo. p, significância estatística dos agrupamentos, pelo teste de permutação de Monte Carlo. 


\section{Conclusões}

1. A abundância e riqueza de espécies dos grupos taxonômicos da macrofauna edáfica varia com a época de coleta, e são favorecidas na época chuvosa.

2. Os sistemas de manejo e de preparo do solo afetam a estrutura dos grupos taxonômicos dominantes da macrofauna edáfica.

3. Os sistemas agroflorestais propiciam melhores características químicas do solo e aumentos na abundância e riqueza de espécies da macrofauna invertebrada do solo.

\section{Agradecimentos}

À Coordenação de Aperfeiçoamento de Pessoal de Nível Superior e a Empresa Brasileira de Pesquisa Agropecuária, pelo auxílio financeiro e logístico.

\section{Referências}

ALVES, M.V.; BARETTA, D.; CARDOSO, E.J.B.N. Fauna edáfica em diferentes sistemas de cultivo no estado de São Paulo. Revista de Ciências Agroveterinárias, v.5, p.33-43, 2006.

ANDERSON, J.M. Why should we care about soil fauna? Pesquisa Agropecuária Brasileira, v.44, p.835-842, 2009.

ANDERSON, J.M.; INGRAM, J.S.I. (Ed.). Tropical soil biology and fertility: a handbook of methods. $2^{\text {nd }}$ ed. Wallingford: CAB International, 1993. 221p.

AQUINO, A.M. de; CORREIA, M.E.F.; ALVES, M.V. Diversidade da macrofauna edáfica no Brasil. In: MOREIRA, F.M.S.; SIQUEIRA, J.O.; BRUSSAARD, L. (Ed.). Biodiversidade do solo em ecossistemas brasileiros. Lavras: UFLA, 2008a. p.143170.

AQUINO, A.M. de; SILVA, R.F. da; MERCANTE, F.M.; CORREIA, M.E.F.; GUIMARÃES, M. de F.; LAVELlE, P. Invertebrate soil macrofauna under different ground cover plants in the no-till system in the Cerrado. European Journal of Soil Biology, v.44, p.191-197, 2008b.

BARROS, E.; MAHIEU, J.; TAPIA-CORAL, S.; NASCIMENTO, A.R.L.; LAVELLE, P. Comunidade da macrofauna do solo na Amazônia brasileira. In: MOREIRA, F.M.S.; SIQUEIRA, J.O.; BRUSSAARD, L. (Ed.). Biodiversidade do solo em ecossistemas brasileiros. Lavras: UFLA, 2008. p.171-191.

BARROS, E.; PASHANASI, B.; CONSTANTINO, R.; LAVELLE, P. Effects of land-use system on the soil macrofauna in western Brazilian Amazonia. Biology and Fertility of Soils, v.35, p.338347,2002

CLAESSEN, M.E.C. (Org.). Manual de métodos de análise de solo. 2.ed. rev. atual. Rio de Janeiro: Embrapa-CNPS, 1997. 212p. (Embrapa-CNPS. Documentos, 1).
COIMBRA, J.L.M.; SANTOS, J.C.P.; ALVES, M.V.; BARZOTTO, I. Técnicas multivariadas aplicadas ao estudo da fauna do solo: contrastes multivariados e análise canônica discriminante. Ceres, v.54, p.270-276, 2007.

CORREIA, M.E.F.; ANDRADE, A.G. Formação da serapilheira e ciclagem de nutrientes. In: SANTOS, G. de A.; SILVA, L.S. da; CANELLAS, L.P.; CAMARGO, F.A.O. (Ed.). Fundamentos da matéria orgânica do solo: ecossistemas tropicais e subtropicais. $2^{\text {nd }}$ ed. Porto Alegre: Metrópole, 2008. p.137-158.

DECAËNS, T.; BUREAU, F.; MARGERIE, P. Earthworm communities in a wet agricultural landscape of the Seine Valley (Upper Normandy, France). Pedobiologia, v.47, p.479-489, 2003.

DIAS, P.F.; SOUTO, S.M.; CORREIA, M.E.F.; ROCHA, G.P.; MOREIRA, J.F.; RODRIGUES, K. de M.; FRANCO, A.A. Árvores fixadoras de nitrogênio e macrofauna do solo em pastagem de híbrido de Digitaria. Pesquisa Agropecuária Brasileira, v.41, p.1015-1021, 2006.

FERREIRA, D.F. SISVAR: um programa para análises e ensino de estatística. Revista Symposium, v.6, p.36-41, 2008.

HÖFER, H; HANAGARTH, W.; GARCIA, M.; MARTIUS, C.; FRANKLIN, E.; RÖMBKE, J.; BECK, L. Structure and function of soil fauna communities in Amazonian anthropogenic and natural ecosystems. European Journal of Soil Biology, v.37, p.229-235, 2001.

LAVELLE, P.; BIGNELL, D.; LEPAGE, M.; WOLTERS, V.; ROGER, P.; INESON, P.; HEAL, OW.; DHILLION, S. Soil function in a changing world: the role of invertebrate ecosystem engineers. European Journal of Soil Biology, v.33, p.159-193, 1997.

LUIZÃO, F.J.; SCHUBART, H.O.R. Litter production and decomposition in a terra-firme forest of central Amazonia. Experientia, v.43, p.259-265, 1987.

MARCHÃO, R.L. Integração lavoura-pecuária num latossolo do cerrado: impacto na física, matéria orgânica e macrofauna. 2007. 153p. Tese (Doutorado) - Universidade Federal de Goiás, Goiânia.

MARCHÃO, R.L.; LAVELLE, P.; CELINI, L.; BALBINO, L.C.; VILELA, L.; BECQUER, T. Soil macrofauna under integrated crop-livestock systems in a Brazilian Cerrado Ferralsol. Pesquisa Agropecuária Brasileira, v.44, p.1011-1020, 2009.

MERLIM, A. de O.; GUERRA, J.G.M.; JUNQUEIRA, R.M.; AQUINO, A.M. de. Soil macrofauna in cover crops of figs grown under organic management. Scientia Agricola, v.62, p.57-61, 2005.

NUNES, L.A.P.L.; ARAÚJO FILHO, J.A. de; MENEZES, R.I. de Q. Diversidade da fauna edáfica em solos submetidos a diferentes sistemas de manejo no semi-árido nordestino. Scientia Agraria, v.10, p.43-49, 2009.

NUNES, L.A.P.L.; ARAÚJO FILHO, J.A. de; MENEZES, R.I. de Q. Recolonização da fauna edáfica em áreas de Caatinga submetidas a queimadas. Caatinga, v.21, p.214-220, 2008.

SANTOS, G.G.; SILVEIRA, P.M. da; MARCHÃO, R.L.; BECQUER, T.; BALBINO, L.C. Macrofauna edáfica associada a plantas de cobertura em plantio direto em um Latossolo Vermelho 
do Cerrado. Pesquisa Agropecuária Brasileira, v.43, p.115-122, 2008.

SANTOS, H.G. dos; JACOMINE, P.K.T.; ANJOS, L.H.C. dos; OLIVEIRA, V.A. de; OLIVEIRA, J.B. de; COELHO, M.R.; LUMBRERAS, J.F.; CUNHA, T.J.F. (Ed.). Sistema brasileiro de classificação de solos. 2.ed. Rio de Janeiro: Embrapa Solos, 2006. $306 \mathrm{p}$.

SILVA, R.F. da; AQUINO, A.M. de; MERCANTE, F.M.; GUIMARÃES, M. de F. Macrofauna invertebrada do solo em sistema integrado de produção agropecuária no Cerrado. Acta Scientiarum Agronomy, v.30, p.725-731, 2008.

SILVA, R.F. da; AQUINO, A.M. de; MERCANTE, F.M.; GUIMARÃES, M. de F. Macrofauna invertebrada do solo sob diferentes sistemas de produção em Latossolo da Região do Cerrado.

Pesquisa Agropecuária Brasileira, v.41, p.697-704, 2006.

SILVA, R.F. da; TOMAZI, M.; PEZARICO, C.R.; AQUINO, A.M. de; MERCANTE, F.M. Macrofauna invertebrada edáfica em cultivo de mandioca sob sistemas de cobertura do solo. Pesquisa Agropecuária Brasileira, v.42, p.865-871, 2007.

TER BRAAK, C.J.F. Ordination. In: JONGMAN, R.H.G.; TER BRAAK, C.J.F.; TONGEREN, O.F.R. van (Ed.). Data analysis in community and landscape ecology. Cambridge: Cambridge University Press, 1995. p.91-173.

THIOULOUSE, J.; CHESSEL, D.; DOLE'DEC, S.; OLIVIER, J.M. ADE-4: a multivariate analysis and graphical display software. Statistics and Computing, v.7, p.75-83, 1997.

VELÁSQUEZ, E.; LAVELlE, P.; ANDRADE, M. GISQ, a multifunctional indicator of soil quality. Soil Biology and Biochemistry, v.39, p.3066-3080, 2007.

VOHLAND, K.; SCHROTH, G. Distribution patterns of the litter macrofauna in agroforestry and monoculture plantations in central Amazonia as affected by plant species and management. Applied Soil Ecology, v.13, p.57-68, 1999.

WOLTERS, V. Invertebrate control of soil organic matter stability. Biology and Fertility of Soils, v.31, p.1-19, 2000.

Recebido em 19 de março de 2009 e aprovado em 3 de fevereiro de 2010 